\title{
Radiaciones ionizantes: Frontera del conocimiento y retos tecnológicos
}

Recibido: 15 Febrero 2014 - Revisado: 30 Abril 2014

Aceptado: 30 Mayo 2014 - Publicado: 30 Julio 2014

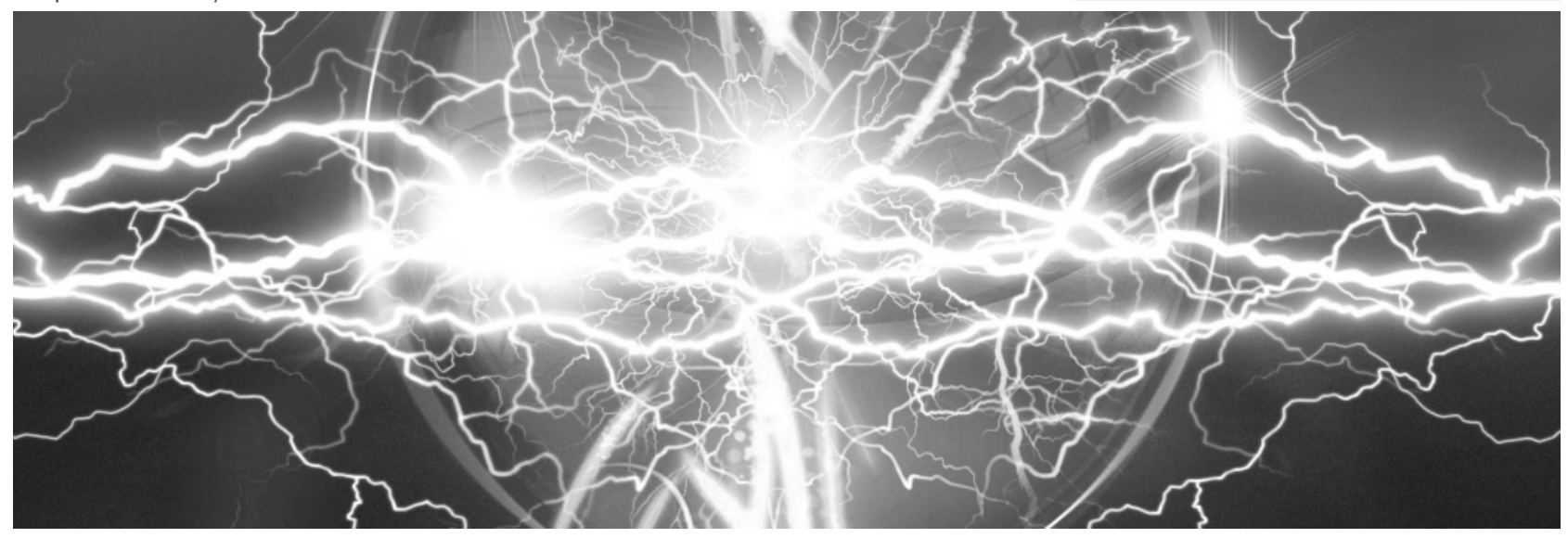

Resumen: El presente trabajo hace referencia al papel de las radiaciones ionizantes en la naturaleza y se examinan sus principales aplicaciones: obtención de energía, medicina nuclear, manejo de recursos hídricos y desarrollo de fármacos. Se indican los avances, así como los retos que deben enfrentar su uso, en particular, los retos tecnológicos para desarrollar la energética nuclear convencional y la basada en la reacción termonuclear controlada. Se presta atención a las modalidades de imagen en medicina nuclear, el papel de los equipos que combinan buena resolución anatómica con sensibilidad, como son Tomografía Axial Computarizada/Tomografía de Emisión de Positrones en Imagen Molecular, el uso de radiofármacos en terapia radionuclídica; ambos como parte de la Medicina Molecular dirigida a enfoques individualizados de atención. Buena parte de los ejemplos se refieren a trabajos desarrollados en Cuba. Finalmente, se reflexiona que la ciencia ha de dar respuesta a los retos encaminando las correspondientes tecnologías. Se considera que el reto principal está en romper la barrera psicológica que impide al hombre vivir en paz, cuidar de su planeta, distribuir equitativamente el fruto de su trabajo.

Palabras clave: Radiaciones Ionizantes, radioisótopos, energía nuclear, Medicina Nuclear, radiofármacos.

Abstract: This paper refers to the role of ionizing radiation in nature and its main applications are discussed: energy production, nuclear medicine, water resources management and development of drugs. The progress and the challenges facing their use, particularly in technology to develop nuclear energy and conventional basis of controlled thermonuclear reaction are indicated. Attention to imaging modalities in nuclear medicine, the role of computers that combine good anatomical resolution sensitivity such as Computed Tomography / Positron Emission Tomography in Molecular Imaging, the use of radiopharmaceuticals in radionuclidic therapy is provided, both as part Molecular Medicine individualized attention directed approaches. Many of the examples relate to work done in Cuba. Finally, we reflect that science has to respond to the challenges related technologies routing. It is considered that the main challenge is to break the psychological barrier that prevents man to live in peace, take care of your planet, equitably distribute the fruits of their labor.

Key words: Ionizing radiations, radioisotopes, nuclear energy, Nuclear Medicine, radiopharmaceuticals. 


\section{INTRODUCCIÓN}

La globalización, proceso característico de nuestra época, amplía tanto la brecha entre desarrollo y no desarrollo, como acentúa los problemas globales que afectan a todos, ricos y pobres; explosión demográfica, escasez de recursos energéticos, deterioro de la naturaleza. Las posibles soluciones no están a la mano y conviene, en cada caso, seleccionar las mejores opciones a fin de acercar la brecha, preservar el patrimonio social y hacer que el hombre sea cada vez más pleno. A ello no son ajenas las ciencias nucleares.

Vivimos en un mundo de radiaciones. La emisión radiactiva de los minerales de uranio y torio contribuye a la temperatura del planeta. La radiación solar está en la base de las transformaciones de los compuestos inorgánicos en orgánicos y en el complicado y sorprendente proceso que ocurre en microorganismos y plantas verdes, la fotosíntesis. Los frutos desdoblados después por el ser vivo animal aportan la energía con la que este realiza los recambios de su sistema, por un infinito número de reacciones bioquímicas, que aseguran eso a la temperatura del cuerpo, 37 grados.

Del abismal cosmos nos llegan partículas cuya esencia no hemos podido aún desentrañar. La ciencia, la indagación, ese proceso que hace al hombre extraer cada vez del infinito mar de la naturaleza, apenas una gota de saber, es la que nos revela que vivimos en un mundo de partículas y ondas, de radiaciones. Objetos de estudio o instrumentos de transformación de la realidad, las radiaciones son parte de la agenda cotidiana de la sociedad humana, obligada continuamente a reflexionar sobre sus beneficios y riesgos (Morín, 2006).

\section{ANTECEDENTES}

\section{La energía del núcleo atómico}

A escala global, el incremento sistemático del consumo energético se considera rasgo de desarrollo. Nos alumbramos con lámparas eléctricas, nos trasladamos en vehículos a motor, preparamos la tierra con tractores, recogemos las cosechas con máquinas, cocinamos con gas, electricidad, carbón, producimos bienes de uso doméstico en fábricas consumidoras de energía.

La población del planeta crece continuamente y cada vez queremos vivir mejor. Este proceso tiene como consecuencia el agotamiento de las fuentes de energía, valoraciones sobre su costo, el daño que causan y los conflictos bélicos que provocan. Hay, pues, necesidad de encontrar caminos para un uso racional y válido de los recursos energéticos.

Una de las fuentes más controvertidas ha sido la que se basa en la energía nuclear, por su vínculo con la carrera armamentista, los accidentes nucleares, la sombra de Hiroshima y Nagasaki y sus secuelas. ¿Debemos, por ello, renunciar a esta forma de energía o perfeccionar su uso? Tal pregunta pudiera también hacerse, como es sabido, en relación con el consumo de combustibles fósiles, causa de la emisión de gases de efecto invernadero, vinculada al calentamiento global y al cambio climático (OIEA, 2006).

La Organización de Naciones Unidas aboga por el uso de fuentes de energías sostenibles y limpias como medida para preservar la naturaleza y asegurar el futuro de la humanidad (ONU, 2012). Es lo que todo ser racional desea, pero el asunto no es sencillo, son muchos los obstáculos y dramáticas, las realidades. Necesitamos cada vez más energía. 
Aunque hay puntos de vista contrarios por considerar adecuados en perspectiva la energía nuclear (ONU, 2011), los pronósticos del Organismo Internacional de Energía Atómica (OIEA) indican, sin embargo, su presencia sostenida: a finales del 2012, se encontraban en operación en todo el mundo, 437 reactores nucleares de potencia y 67 nuevos en construcción (ONU, 2011). Los efectos del accidente de Fukushima Daiichi se siguieron sintiendo en 2012. Para esa época, se enlentece la expansión de la energía nucleoeléctrica, aunque las proyecciones del OIEA indican un importante crecimiento de su uso centrado principalmente en Asia y en los países que ya tienen centrales nucleares en explotación. Es justamente en Asia donde se encuentran 47 de los 67 reactores en construcción (Vilches, 2011).

Los retos principales de esta fuente son su costo, el agotamiento del uranio en el actual ciclo de combustible, la gestión de los desechos radiactivos y la seguridad de las centrales. Aunque constituye solo el $7 \%$ entre todas las fuentes utilizadas en la actualidad, la realidad indica que no se podrá prescindir de ella, al menos al corto plazo, pues se avizoran soluciones a esas dificultades, en particular al problema de los desechos radiactivos, en un escenario de crecimiento sostenido del consumo (ONU, 2011; Cano, 2008; Milaniev, 1987). El OIEA ha desarrollado un grupo de acciones al respecto como es el Proyecto Internacional sobre ciclos del combustible y reactores nucleares innovadores (Inpro), que presta apoyo a los Estados Miembros en el desarrollo y la utilización de sistemas de energía nuclear sostenibles (Vilches, 2011).

El factor tecnológico tiene en este campo, pues, un reto indiscutible y ha de contribuir sin dudas a encontrar respuestas efectivas. En materia de energía nuclear a más largo plazo, son de singular interés los trabajos desarrollados por décadas para llegar a controlar la energía liberada durante la fusión nuclear, fuente de la energía solar, opción energética basada en recursos prácticamente inagotables, agua en esencia, y con escasa influencia medioambiental.

La fusión nuclear consiste en la unión de dos núcleos atómicos para obtener un núcleo de mayor tamaño, con la liberación de energía. Para acercar dos núcleos de carga positiva, hay que superar la fuerza de repulsión electrostática, es decir, suministrar energía al sistema. El proceso desarrollado consiste en calentar a grandes temperaturas una mezcla de dos isótopos de hidrógeno (los isótopos son átomos de un mismo elemento con diferentes masas), deuterio y tritio (con un solo protón en su núcleo para que la repulsión sea mínima); llevarlos a un estado en el que los electrones se separan del átomo, se forman iones positivos y se tiene un gas altamente ionizado, el plasma. Debido a sus notables propiedades físicas, muy distintas a las de los gases, líquidos y sólidos, el plasma es considerado el "cuarto estado de la materia". Las estrellas, el Sol entre ellas, están compuestas por plasma. La energía emitida por el Sol se debe a reacciones de fusión a enormes presiones y temperaturas que existen en su interior (Bishop, 2013).

Una de las principales dificultades a la hora de producir la fusión ha sido cómo poder confinar el plasma a temperaturas de millones de grados, otra que adquiera gran densidad. La reacción termonuclear y la liberación de energía tienen lugar como resultados de los choques entre sí de los núcleos de deuterio y tritio. El número de tales choques será tanto mayor cuanto mayor sea el número de partículas por centímetro cúbico. Para que la reacción produzca un rendimiento positivo de energía, o sea, que la energía que se libera sea superior a los gastos energéticos vinculados a la obtención y calentamiento del plasma, la reacción deberá prolongase un tiempo lo suficientemente largo. 
Conocemos dos dispositivos en los cuales se cumplen estas exigencias: la bomba termonuclear y el Sol. En este reactor natural, por medio de las fuerzas de gravedad, se resuelve el problema más serio para los físicos: el de la conservación, el confinamiento del plasma caliente. Como en el estado de plasma las partículas están ionizadas, es decir, con carga, pueden ser confinadas usando campos magnéticos. Es una de las vías que se han seguido (Bishop, 2013). El confinamiento evita la interacción del plasma con los materiales del dispositivo, del reactor de fusión.

Los experimentos realizados tienden a asegurar que las reacciones de fusión produzcan más energía que la que consumen y, en perspectiva, que pueda utilizarse de manera controlada para producir electricidad. El Proyecto ITER, (International Thermonuclear Experimental Reactor: Reactor Termonuclear Experimental Internacional) está destinado a probar que la tecnología de fusión nuclear es viable. Se basa en un reactor de confinamiento magnético (Tokamak), que se construye en Cadarache (Francia). En el Tokamak, el plasma alcanza una temperatura elevada por el calentamiento producido por la inmensa corriente inducida por el campo magnético en forma de toroide, parecida a la de una rosquilla.

Los países están desarrollando sus propias estrategias de manera independiente, como la de confinamiento inercial mediante calentamiento de una cápsula pequeña llena de deuterio y tritio con láseres potentes; recientemente, se indicó la obtención de ganancia de energía en el proceso (OIEA, 2003). Se estima que dada la amplitud de conocimientos especializados y la escala de las instalaciones y actividades necesarias para el desarrollo de la fusión, la colaboración internacional continuará siendo un componente esencial para progresar en esta esfera (Vilches, 2011). Se prevé la tecnología pueda estar lista en unos 40 años y que por su elevado costo, sea en particular privativa de los países.

Hidrología isotópica
El agua de la tierra viene de las precipitaciones, se filtra por el suelo para formar las aguas subterráneas o se escurre y va a ríos y lagos, y al mar finalmente. Toda la superficie expuesta se evapora, para precipitar de nuevo y cerrar ese ciclo. Solo el 2,5\% del agua de la Tierra es dulce. La mayor parte está en forma de hielo polar, o humedad del suelo, o a profundidades inaccesibles. Queda disponible, para el uso, menos del $1 \%$.

El quehacer humano afecta no solo la cantidad de ese precioso recurso, sino su calidad. Ante la amenaza cada vez mayor de una creciente escasez de agua, las decisiones sobre los lugares donde debe extraerse, la cuantía que ha utilizarse y la manera en que se debe suministrar tienen que basarse en información confiable. Hay que cuidar también que el agua no pierda sus virtudes, evitar su deterioro. El desarrollo humano sostenible depende de la disponibilidad de agua (Stosic, 1961). En la antigüedad, el arte del manejo del agua se consideraba, de una u otra manera, la llave del progreso (Aggarwall, 2007).

Desde que se usaron briznas para averiguar el nacimiento del río Jordán, hace ya un par de milenios, la introducción de trazadores capaces de indicar de donde vienen, cómo se mueven, hacia dónde van las aguas, se ha hecho práctica habitual en hidrología.

La hidrología isotópica se ocupa de las aplicaciones de los isótopos como trazadores en el desarrollo y manejo de los recursos hídricos. Se basa en el uso de isótopos presentes en el ambiente o de isótopos artificiales. Los primeros permiten estudiar distintos procesos en una escala espacial y temporal mayor, mediante la evaluación de su distribución natural en un sistema hidrológico. Los trazadores artificiales son efectivos en aplicaciones locales en un sitio específico (Domínguez, 1998).

Una peculiar aplicación ha sido la de utilizar Tecnecio99m, empleado en medicina nuclear diagnóstica, para evaluar la cuenca del río Almendares en La 
Habana (Borroto, 2003). Este trazador vive poco, cada seis horas desaparece la mitad de los átomos presentes, se mide bien y es muy versátil desde el punto de vista químico. Se pudo comprobar que el Tecnecio-99m se comporta de forma similar a un colorante muy usado en aguas superficiales contaminadas y simula el comportamiento de los residuales.

Sedimentos marcados con este radionúclido responden adecuadamente a las características hidromorfológicas en corrientes superficiales. Así, el Tecnecio-99m puede usarse como radiotrazador para el marcaje de aguas naturales y residuales y sedimentos en estudios hidrológicos, hidrodinámicos y sedimentológicos a corto plazo (Phelps, 2000).

\section{METOdOLOGÍA}

\section{Medicina Nuclear e Imagen Molecular}

Una imagen médica es la representación visual de objetos o procesos relacionados con el cuerpo humano. De acuerdo a como sea obtenida, tendremos: imagen óptica, de rayos $X$, nuclear, ultrasónica o de resonancia magnética. La Imagen Nuclear brinda de manera no invasiva, información funcional a niveles celular y molecular, mediante la medición de la captación y movimiento de trazadores radiactivos de blancos específicos en los tejidos.

Se utilizan dos tecnologías: La Tomografía Computarizada por Emisión de Fotón Único (SPECT) y la Tomografía por Emisión de Positrones (PET). LOS trazadores radiactivos en medicina nuclear reciben el nombre de radiofármacos, término que incluye no solo emisores de fotones, base de las dos tecnologías de imagen nuclear diagnóstica indicadas, sino emisores de radiación beta, es decir, electrones rápidos, cuando el fin es curativo (terapia radionuclídica).
Como las enfermedades resultan de la introducción de defectos en los sistemas bioquímicos del organismo por medio de virus, bacterias, anormalidades genéticas y drogas o por efecto del envejecimiento, de los patrones de conducta o del medio ambiente (Thakur, 2006); la detección de señales biológicas a nivel tisular y celular asociadas a una molécula específica explican el interés de los avances en Imagen Médica, en particular en Imagen Nuclear.

Esto se debe a su elevada sensibilidad para visualizar procesos moleculares en comparación con Tomografía Axial Computarizada (TAC), Ecografía o Imagen de Resonancia Magnética (IRM); aunque esta última y la imagen óptica son hoy parte esencial de la Imagen Molecular. Las técnicas de Imagen Molecular monitorean y registran directa o indirectamente la distribución espacio-temporal de procesos celulares o moleculares para aplicaciones bioquímicas y biológicas tanto diagnósticas como terapéuticas (Zaidi, 2006).

La obtención de información de anormalidades antes de la expresión clínica y su corrección al nivel más básico inicial sustenta la medicina molecular. La Imagen Molecular desempeña así un importante papel en la evaluación de dianas celulares y su respuesta a terapia en diagnóstico diferencial, en la selección de pacientes que pueden beneficiarse de un determinado tratamiento y en la dosimetría para terapia de diana (Townsend, 2004); es decir, al diagnóstico temprano que conduce a terapia molecular individualizada y orientada también por la imagen.

Los avances tecnológicos del equipamiento de imagen y el desarrollo de nuevos radiofármacos con base a péptidos, anticuerpos monoclonales, es la línea de acción en Medicina Nuclear para el diagnóstico temprano y el tratamiento del cáncer y otras patologías. Sirva de ejemplo la combinación en 
un solo equipo de la información funcional de SPECT y, sobre todo, de PET con la mejor delimitación anatómica de TAC, lo que favorece el diagnóstico y la terapia radiacional (Young, 2013).

Con ayuda de un nuevo radiofármaco para PET, se ha detectado aumento en el depósito de beta amiloide en regiones del cerebro, luego de daño cerebral postraumático, lo que puede contribuir al manejo de estos incidentes, a la posibilidad de identificar pacientes con alto riesgo de enfermedad de Alzheimer acelerada y evaluar los beneficios potenciales de la terapia antiamiloide (Leyva \& Morín, 2012).

En terapia radionuclídica, se han introducido radiofármacos útiles en el tratamiento del Linfoma no Hodgkin y tumores de origen neuroendocrino, entre otros (OIEA, 2006). Se desarrollan también radiofármacos basados en anticuerpos monoclonales (Leyva \& Morín, 2012). En Cuba se reportan excelentes resultados con una nueva formulación de Fosfato de Cromo (III) marcado con el beta emisor 32P en el tratamiento de sinovitis crónica en pacientes artríticos y hemofílicos (Cruz, 2013).

\section{Imagen Nucleary desarrollo de fármacos}

Las empresas farmacéuticas dedican cuantiosos recursos a la búsqueda continua de moléculas líderes. Utilizan, para ello, métodos de modelación, tamizaje in vitro y estudios animales. El progreso de la Imagen Molecular ha probado ser eficaz en abaratar los costos de esas investigaciones y se ha convertido en paso casi obligado antes de pasar a la fase clínica de investigación sobre la base que moléculas con muy buenas características, reveladas por otros métodos, han resultado después ineficaces.

En este enfoque, la Imagen Nuclear, como parte de la Imagen Molecular, tiene un destacado papel. Además, el empleo de trazadores radiactivos aporta valiosísima información no clínica, necesaria para el registro de los productos de interés, por los órganos regulatorios. El desarrollo en Cuba de fármacos, tanto a partir de fuentes naturales como por los métodos de la Ingeniería Genética y la Inmunología Molecular, ha requerido de estudios no clínicos con trazadores, incluida la Imagen Molecular.

La explicación es sencilla: estas técnicas pueden reducir en un tercio el tiempo de ensayo en las fases I y Il de aprobación de un fármaco, con la disminución del costo en el proceso de investigación (Hernández, 2015). En esta reciente revisión que citamos, se hace referencia al estudio de absorción percutánea in vivo en ratas e in vitro en celdas de difusión de melagenina marcada con 125l, con el objetivo de probar que el producto, de uso tópico, no se incorporaba al lecho vascular, factor que contribuyó al registro médico de este fármaco cubano eficaz en el tratamiento del vitíligo.

Se indica, asimismo, la obtención del perfil farmacocinético de una formulación del anticuerpo monoclonal humanizado hR3 y la preparación de radiofármacos para diagnóstico y terapia de cáncer, marcados con 99mTc,186Re e 90Y con base también a productos asociados al receptor del factor de crecimiento epidérmico (EGF-r) blanco celular de interés en Cuba desde hace varios años (Leyva \& Morín, 2012; Hernandez, 2015).

Un producto de esta línea, el Heberprot, de aplicación local en ulceraciones de extremidades en pacientes diabéticos ha permitido implementar un tratamiento efectivo ante esta complicación, que conduce frecuentemente a la amputación del miembro. Bien probada la utilidad clínica de Heberprot, quedaban factores por caracterizar desde el punto de vista experimental. El marcaje del producto con $125 \mathrm{I}$ se realizó mediante el método del iodógeno, la purificación por exclusión molecular, los estudios de farmacocinética y biodistribución en ratones y ratas después de una administración y a dosis repetidas (Hernández, 2015). 


\section{CONCLUSIONES}

Las radiaciones ionizantes continuarán desempeñando un papel primordial en el progreso humano reflejado en la producción de energía, gestión de recursos hídricos, salud, entre otras áreas; aunque enfrentan para ello, importantes retos cognoscitivos y tecnológicos. Es presumible, no obstante, que sean resueltas las dificultades para obtener energía de fusión mediante reacciones termonucleares controladas, que la acción terapéutica pueda diseñarse de forma individualizada, que nos acerquemos cada vez más al conocimiento de los fenómenos cósmicos. Nos las arreglaremos también para asegurarnos el agua.

La Tierra, como cuerpo celeste, su riqueza mineral, vegetal, animal y social es un organismo vivo. No comprender eso es a todas luces la principal barrera al progreso verdadero. Persiste hasta ahora la división entre ricos y pobres, no cesan las guerras, el planeta muere. La propiedad fundamental de la ciencia es la presencia de determinadas leyes a las que están sujetos fenómenos en todas sus partes componentes. Hay que aplicarla al planeta como conjunto: hacer uso de sus recursos sin comprometer su destino, es el verdadero reto tecnológico.

\section{REFERENCIAS}

Aggarwal, P.K., Froehlich, K., Kulkarni, K.M. (2007). Environmental Isotopes in Ground water Studies eolss. Recuperado de: http://www.eolss.net/SampleChapters/C07/E2- 09-03-06.pdf

Asamblea General las Naciones Unidas (2011). Recuperado de:

http://www.um.es/prinum/rn12/files/2012_A\%C3\% B1o_Internacional_Energia_Sostenible_Declaracion. pdf

Asamblea General de Naciones Unidas (2012). Resolución 65/151. Año Internacional de la Energía Sostenible para Todos. Recuperado de: http://www.un.org/es/events/sustainableenergyfora II/

Bishop, B. (2013). Laser fusion experiments yields record energy at Lawrence Livermore's National Ignition Facility. Recuperado de:

https://www.Ilnl.gov/news/newsreleases/2013/Aug/ NR-13-08-04.html.

Borroto, J. (2003). Comportamiento del Tc99m como radiotrazador en estudios hidrológicos a corto plazo. Tesis en opción al grado de Doctor en Ciencias Químicas, Ciudad de la Habana, Cuba.

Cano, D. (2008). Revista Española de Física, EneroMarzo. Recuperado de: http://www.rsef.org

Comitee on the State of Sciences of Nuclear Medicine Advancing Nuclear Medicine (2007). Through Innovation. Washington D.C., USA: The National Academic Press.

Cruz Arencibia J. (2013). Fosfato de Cromo (III) marcado con diferentes radionúclidos para uso en Radiosinoviortesis, Tesis en opción al título de Doctor en Ciencias Farmacéuticas. La Habana, Cuba. 
Domínguez, J., Borroto, J., Abreu, A. M., Ortueta M., Pérez, E. (1998). "Medición de caudal en el río Almendares empleando 99mTc como radiotrazador». Serie Contribución a la Educación y Protección Ambiental. Hombre y Medio Ambiente, 173. Habana, Cuba: Editorial Academia.

Hernández I. (2015). «Investigaciones no clínicas en el Centro de Isótopos en función de la industria farmacéutica y biotecnológica». Nucleus 52(35).

H. Zaidi. (2006). "The quest for the ideal anatomolecular imaging fusion tool». Biomed Imaging Interv J. 2(4).

IAEA, (2006). Informe del Director General, G.C. Examen de la tecnología nuclear. (50)/INF/3.

International Atomic Energy Agency (2003). Managing Water Resources using Isotope Hydrology, International Atomic Energy Agency. Information Series. Recuperado de:

http://www.iaea.org/sites/default/files/water.pdf

Leyva, R., Perera, A., Morín, J.A. (2012). «Radiofármacos en inmunocentelleografía y Radioinmunoterapia». Nucleus 52(68).

Milántiev V., Temkó S. Física del plasma. Colección Física al alcance de todos, editorial Mir (1987).

Morín, J.A. (2006). «Pertinencia y riesgo de las aplicaciones nucleares». Nucleus, 40(4).

Phelps, M.E., (2000). «Positron emission tomography provides molecular imaging of biological processes». PNAS 97 (16), pp. 9226.

Stosic, Borko D. (1961). Use of isotopes in hydrologyInternational Atomic Energy Agency. Recuperado de: http://www.iaea.org/Publications/Magazines/Bulleti n/Bull042/04205902124.pdf
Thakur, M., Lentle, B.C. (2006). Report of a Summit on Molecular Imaging.Department of Radiology, Division of Nuclear Medicine, Thomas Jefferson University Medical College. Ajronline.

Townsend, D.W., Carney, J.P., Yap JT, et al. (2004). «PET/CT today and tomorrow». Nucl Med J. 45 Suppl 1,4S-14S.

Vilches, G. \& Pérez, D. (2011). Año Internacional de la Energía Sostenible para todos: La transición de las energías no renovables a la energía sostenible. Revista Española de Física, 26 (4).

Young T. Hong et al. (2013). «Amyloid imaging with carbon 11-labeled Pittsburgh compound B for traumatic brain injury». JAMA Neurol. 71 (1), 23-31.

\section{APÉNDICE A}

\section{Retos del reactor termonuclear}

La reacción termonuclear controlada con rendimiento positivo de energía necesita tres condiciones:

1. Una mezcla de los isótopos pesados del hidrógeno deuterio y tritio debe ser calentada hasta una temperatura de 100 millones de grados centígrados.

2. La mezcla caliente de deuterio y tritio debe poseer una densidad lo suficientemente grande.

3. Para que la reacción produzca un rendimiento positivo de energía, o sea, que la energía que se libere sea superior a los gastos energéticos vinculados a la obtención y calentamiento del plasma, la reacción deberá prolongase un tiempo lo suficientemente largo.

Las Técnicas Isotópicas son efectivas en (Stosic, 1961):

- Aportar información valiosa sobre el origen de las aguas subterráneas. 
- La determinación de la edad, velocidad y dirección del flujo de estas.

- Establecer las posibles interconexiones entre aguas subterráneas y superficiales.

- Caracterizar los acuíferos.

Aplicaciones rutinarias de la Medicina Nuclear (Hernández, 2015)

- Diagnóstico de cáncer, desórdenes neurológicos como enfermedad de Parkinson y Alzheimer, estado inicial de enfermedad cardiovascular con reducción de morbilidad y mortalidad.

- Evaluación no invasiva de la respuesta terapéutica; se reduce la exposición de los pacientes a la acción tóxica de terapias no efectivas y permite incorporar más temprano otras alternativas.

- Tratamiento dirigido a blancos moleculares en cáncer y desórdenes endocrinos (enfermedad tiroidea, tumores de origen neuroendocrino, otros).

- Oportunidades emergentes en Medicina Nuclear (Hernández, 2015)

- Comprensión de la relación entre química cerebral y conducta.

- Estimación de la aterosclerosis del sistema cardiovascular.

- Comprensión del metabolismo y farmacología de nuevos medicamentos.

- Evaluar la eficacia de nuevos fármacos, acelerando su introducción en la práctica clínica.

- Empleo de la terapia radionuclídica para individualizar el tratamiento de pacientes con cáncer mediante selección de las propiedades del vehículo orientado al blanco y del radionúclido.

- Desarrollo ulterior y explotación de los sistemas de imagen híbridos como PET/RMN, para mejorar el diagnóstico y el tratamiento de enfermedades.

- Mejorar la producción de radionúclidos y aumentar la disponibilidad de radiofármacos. 